\title{
Variations in Pumpkin (Cucurbita moschata Duch. ex Poir.) Landraces for Earliness, Yield and Quality Attributes in North East India
}

\author{
Md. Ramjan*, A.K. Pandey, Megha Raghavan", \\ Hau Ngaih Lian ${ }^{1}$ and Mohd Talha Ansari ${ }^{1}$ \\ College of Horticulture and Forestry, Central Agricultural University, Pasighat-791102 \\ Arunachal Pradesh, India \\ *Corresponding author
}

\section{A B S T R A C T}

\section{Keywords}

Earliness, Pumpkin, Quality and yield

Article Info

Accepted:

06 June 2018

Available Online:

10 July 2018
The present study was carried out to evaluate the mean performance of pumpkin genotypes for growth, yield and quality attributes. Genotypes were collected from various places of North Eastern Region of India and laid out in Randomized Completely Block Design (RCBD) with three replications. The analysis of variance in the present investigation revealed significant differences among 25 pumpkin genotypes for all the studied traits. On the basis of mean performance, the genotype CHFPUM-24 (Landrace from Tripura) was found good for earliness and growth. The genotype CHFPUM-16 (landrace from Imphal, Manipur) was found to be superior for the yield attributing characters while CHFPUM-1 (landrace from Pasighat, Arunachal Pradesh) was found good for fruit quality. So, these genotypes can be used as parental source in future breeding programme.

\section{Introduction}

Pumpkin (Cucurbita moschata Duch. ex Poir.) is one of the most important cucurbitaceous vegetable crops grown extensively in tropical and sub-tropical parts of the country. Pumpkin is also known as Kashiphal or Sitaphal or Lal Kaddu. It is great significance due to its high carotene content and long shelf life, long period of availability, excellent response to vegetable forcing, high nutritive estimates and better transport qualities. Archeological evidences suggest that Cucurbita moschata was widely distributed in America, Mexico and Peru being the primary center of origin. The name pumpkin originated from Greek word 'Pepon' used for long melon (Bahadur and Singh, 2014).

The flowers are used for excellent preparation so called 'pakora'. The flowers of pumpkin are more nutritive than fruits (Rana 2014). Pumpkin fruits are rich in vitamins, particular in precursor of vitamin $\mathrm{A}$, and minerals. The edible portion of pumpkin constitutes around $80 \%$. Pumpkin seeds contain $40.27 \%$ protein, $34.59 \%$ crude fibre, and 4.4.5\% ash. Oleic, linoleic and palmitic acids are the predominant fatty acid in seed oil. Gulatamic acid, arginine, glycine and aspartic acid are the most abundant amino acids (Aboul-Nasr et el. 1997). The main nutrients are lutein and both 
alpha and beta carotene, the latter of which generates vitamin $\mathrm{A}$ in the body (Ahmed et al., 2011).

Pumpkin has received little attention in crop improvement, as compared to other Cucurbitaceous vegetables. Since ancient times, a wide number of germplasms are available, conscious evaluation and exploitation of germplasm has not been attended until recently. This is very helpful for a plant breeder in developing a commercial variety with market preference by determining the component characters on which selection can be exercised based on the improvement in yield and quality Tamilselvi and Jansirani (2017). Preliminary identification of early maturing genotypes can be done based on characters like days to first opening of staminate flower and female flower, node number to first male and female flowering appearance and days to fruit harvest, number of fruits per plant, average fruit weight and fruit yield per plant.

\section{Materials and Methods}

\section{Experimental material and site}

The present experiment was conducted at Vegetable Research Farm, College of Horticulture and Forestry, Central Agricultural University, Pasighat, East Siang, Arunachal Pradesh, India during March 2017. The experimental material for the present study consisted of 25 diverse genotypes of pumpkin (Cucurbita moschata Duch. ex. Poir.) including two check varieties viz., CHFPUM7 (Kashi Harit) and CHFPUM-23 (Narendra Agrim) were collected from North Eastern Region of India (Table 1).

The experiment was laid out in Randomized complete block design (RCBD) with three replications. Under each genotype 10 plants were sown per replication with spacing of
$3 \times 1.3 \mathrm{~m}^{2}$. The standard cultural practices as mentioned in Package of Practices for Vegetable Crops (Rana. 2014). The observations were recorded from five randomly selected plants for growth, Yield and quality parameters.

\section{Statistical analysis}

Analysis of variance of the data from each attribute was computed by using the OPSTAT computer program. For significant result mean seperation was carried out using least significant difference at 5 per cent level of probability (Gomez and Gomez 1984).

\section{Results and Discussion}

\section{Morphological characters}

The genotypes showed significant variation for all the studied characters. Earliness is considered as desirable traits in cucurbits which is measured as the days taken for first female flower appearance and node number for first female flower appearance. Minimum days taken to first staminate flower anthesis was observed in the genotype CHFPUM-15 (58.53 days) followed by CHFPUM-14 (58.83 days) and CHFPUM-4 (58.87 days) while days taken to first pistillate flower anthesis was observed in CHFPUM-5 (62.00 days) followed by CHFPUM-23 (62.30 days) and CHFPUM-19 (62.53 days) (Table 2). Similar result was reported by Tamilselvi and Jansirani (2017).

Earliness is also measured in node at which first male and female flower appears. Staminate flower first appeared in CHFPUM24 (2.73), followed by CHFPUM- 20 and CHFPUM-21 while first pistillate flower was observed in CHFPUM-24 (10.43) followed by CHFPUM-14 and CHFPUM-20 (Table 2). The result was in conformity with the finding of Venkatesan et al., (2016) in muskmelon. 
Early harvest is also one of the important desirable traits for crop improvement. The pumpkin fruits first matured in genotypes CHFPUM-21 (79.20 days) followed by CHFPUM-23 (81.10 days) and CHFPUM-24 (81.30 days). Similar result was obtained by the (Venkatesan et al., 2016) in muskmelon (Table 2).

\section{Yield contributing characters}

The maximum polar circumference was recorded in CHFPUM-20 (68.93) which was statistically at par with CHFPUM-10,
CHFPUM-23 and CHFPUM-6 (Table 2). The highest equatorial circumference of fruit was obtained from CHFPUM- 23 (73.80) followed by CHFPUM-10 (67.93) and CHFPUM-21 (64.13) whereas lowest was found in CHFPUM-16 (33.93). Similar results were also reported by the (Ahmed et al., 2011) (Table 3). Fruit flesh thickness is a desirable trait in pumpkin and maximum flesh thickness was found in genotypes CHFPUM-12 (5.16) followed by CHFPUM-17(4.76), CHFPUM-3 and CHFPUM-4 (4.50). Similar results reported by the (Tamilselvi and Jansirani, 2017) (Table 3).

Table.1 List of pumpkin genotypes with their sources of collection

\begin{tabular}{|c|c|c|c|}
\hline S. $\mathbf{N}$. & Genotype & Source & Coordinates of the places \\
\hline 1. & CHFPUM-1 & A Landrace of Pasighat, Arunachal Pradesh & $28.07^{\circ} \mathrm{N}, 95.33^{\circ} \mathrm{E}$ \\
\hline 2. & CHFPUM-2 & A landrace of Pasighat, Arunachal Pradesh & $28.07^{\circ} \mathrm{N}, 95.33^{\circ} \mathrm{E}$ \\
\hline 3. & CHFPUM-3 & A landrace of Pasighat, Arunachal Pradesh & $28.07^{\circ} \mathrm{N}, 95.33^{\circ} \mathrm{E}$ \\
\hline 4. & CHFPUM-4 & A landrace of Pasighat, Arunachal Pradesh & $28.07^{\circ} \mathrm{N}, 95.33^{\circ} \mathrm{E}$ \\
\hline 5. & CHFPUM-5 & A landrace of Pasighat, Arunachal Pradesh & $28.07^{\circ} \mathrm{N}, 95.33^{\circ} \mathrm{E}$ \\
\hline 6. & CHFPUM-6 & A landrace of Pasighat, Arunachal Pradesh & $28.07^{\circ} \mathrm{N}, 95.33^{\circ} \mathrm{E}$ \\
\hline 7. & CHFPUM-7 & A landrace of IIVR, Varanasi (U.P.) & $25.28^{\circ} \mathrm{N}, 82.96^{\circ} \mathrm{E}$ \\
\hline 8. & CHFPUM-8 & A landrace of Pasighat, Arunachal Pradesh & $28.07^{\circ} \mathrm{N}, 95.33^{\circ} \mathrm{E}$ \\
\hline 9. & CHFPUM-9 & A landrace of Pasighat, Arunachal Pradesh & $28.07^{\circ} \mathrm{N}, 95.33^{\circ} \mathrm{E}$ \\
\hline 10. & CHFPUM-10 & A landrace of Ziro, Arunachal Pradesh & $27.56^{\circ} \mathrm{N}, 93.83^{\circ} \mathrm{E}$ \\
\hline 11. & CHFPUM-11 & A landrace of Aizwal, Mizoram & $23^{\circ} 43^{\prime} 38^{\prime \prime} \mathrm{N}, 92^{\circ} 43^{\prime} 4^{\prime \prime} \mathrm{E}$ \\
\hline 12. & CHFPUM-12 & A landrace of Aizwal, Mizoram & $23^{\circ} 43^{\prime} 38^{\prime \prime} \mathrm{N}, 92^{\circ} 43^{\prime} 4^{\prime \prime} \mathrm{E}$ \\
\hline 13. & CHFPUM-13 & A landrace of Aizwal, Mizoram & $23^{\circ} 43^{\prime} 38^{\prime \prime} \mathrm{N}, 92^{\circ} 43^{\prime} 4^{\prime \prime} \mathrm{E}$ \\
\hline 14. & CHFPUM-14 & A landrace of Aizwal, Mizoram & $23^{\circ} 43^{\prime} 38^{\prime \prime} \mathrm{N}, 92^{\circ} 43^{\prime} 4^{\prime \prime} \mathrm{E}$ \\
\hline 15. & CHFPUM-15 & A landrace of Imphal, Manipur & $24.80^{\circ} \mathrm{N}, 93.93^{\circ} \mathrm{E}$ \\
\hline 16. & CHFPUM-16 & A landrace of Imphal, Manipur & $24.80^{\circ} \mathrm{N}, 93.93^{\circ} \mathrm{E}$ \\
\hline 17. & CHFPUM-17 & A landrace of Imphal, Manipur & $24.80^{\circ} \mathrm{N}, 93.93^{\circ} \mathrm{E}$ \\
\hline 18. & CHFPUM-18 & A landrace of Gangtok, Sikkim & $27.33^{\circ} \mathrm{N}, 88.62^{\circ} \mathrm{E}$ \\
\hline 19. & CHFPUM-19 & A landrace of Gangtok, Sikkim & $27.33^{\circ} \mathrm{N}, 88.62^{\circ} \mathrm{E}$ \\
\hline 20. & CHFPUM-20 & A landrace of Kohima, Nagaland & $25.67^{\circ} \mathrm{N}, 94.10^{\circ} \mathrm{E}$ \\
\hline 21. & CHFPUM-21 & A landrace of Kohima, Nagaland & $25.67^{\circ} \mathrm{N}, 94.10^{\circ} \mathrm{E}$ \\
\hline 22. & CHFPUM-22 & A landrace of Kohima, Nagaland & $25.67^{\circ} \mathrm{N}, 94.10^{\circ} \mathrm{E}$ \\
\hline 23. & CHFPUM-23 & A landrace of NDUAT, Faizabad (U.P.) & $26.77^{\circ} \mathrm{N}, 82.14^{\circ} \mathrm{E}$ \\
\hline 24. & CHFPUM-24 & A landrace of Agartala, Tripura & $23.84^{\circ} \mathrm{N}, 88.62^{\circ} \mathrm{E}$ \\
\hline 25. & CHFPUM-25 & A landrace of Agartala, Tripura & $23.84^{\circ} \mathrm{N}, 88.62^{\circ} \mathrm{E}$ \\
\hline
\end{tabular}


Table.2 Morphological characters of 25 pumpkin germplasm

\begin{tabular}{|c|c|c|c|c|c|c|c|c|c|c|}
\hline $\begin{array}{l}\text { Sl. } \\
\text { No. }\end{array}$ & Charectes & $\begin{array}{l}\text { Vine } \\
\text { length }\end{array}$ & $\begin{array}{l}\text { Number } \\
\text { of } \\
\text { primary } \\
\text { branches } \\
\text { per plant }\end{array}$ & $\begin{array}{l}\text { Days to } \\
\text { first male } \\
\text { flower } \\
\text { anthesis }\end{array}$ & $\begin{array}{l}\text { Days to } \\
\text { first } \\
\text { female } \\
\text { flower } \\
\text { antheis }\end{array}$ & $\begin{array}{l}\text { Node } \\
\text { bearing } \\
\text { first } \\
\text { male } \\
\text { flower }\end{array}$ & $\begin{array}{l}\text { Node } \\
\text { bearing } \\
\text { first } \\
\text { female } \\
\text { flower }\end{array}$ & $\begin{array}{l}\text { Days to } \\
\text { first } \\
\text { fruits } \\
\text { harvest }\end{array}$ & $\begin{array}{l}\text { Nimber } \\
\text { of fruits } \\
\text { per plant }\end{array}$ & $\begin{array}{l}\text { Polar } \\
\text { circumf } \\
\text { e- } \\
\text { rences }\end{array}$ \\
\hline 1. & CHFPUM-1 & 11.00 & 10.53 & 62.97 & 66.17 & 6.33 & 11.33 & 88.87 & 3.37 & 62.67 \\
\hline 2. & CHFPUM-2 & 7.10 & 13.63 & 63.50 & 70.73 & 5.63 & 12.30 & 103.33 & 3.73 & 52.90 \\
\hline 3. & CHFPUM-3 & 8.10 & 11.97 & 59.10 & 63.40 & 3.73 & 12.10 & 87.60 & 2.90 & 55.10 \\
\hline 4. & CHFPUM-4 & 7.07 & 12.33 & 58.87 & 66.33 & 4.40 & 14.00 & 89.67 & 2.33 & 60.13 \\
\hline 5. & CHFPUM-5 & 6.70 & 9.53 & 60.53 & 62.00 & 5.07 & 12.00 & 86.67 & 2.07 & 50.00 \\
\hline 6. & CHFPUM-6 & 8.50 & 11.10 & 62.07 & 67.73 & 5.00 & 13.60 & 91.43 & 2.87 & 66.10 \\
\hline 7. & CHFPUM-7 & 7.33 & 11.53 & 61.73 & 64.63 & 3.77 & 12.20 & 87.03 & 1.63 & 52.30 \\
\hline 8. & CHFPUM-8 & 7.77 & 8.07 & 61.10 & 63.87 & 3.53 & 13.33 & 87.63 & 2.77 & 41.93 \\
\hline 9. & CHFPUM-9 & 7.40 & 10.97 & 60.73 & 63.53 & 3.73 & 12.10 & 89.10 & 3.13 & 51.00 \\
\hline 10. & CHFPUM-10 & 7.88 & 9.67 & 63.40 & 70.20 & 4.73 & 12.83 & 93.40 & 2.90 & 67.63 \\
\hline 11. & CHFPUM-11 & 7.20 & 11.33 & 63.07 & 69.07 & 4.73 & 12.27 & 93.43 & 2.80 & 57.30 \\
\hline 12. & CHFPUM-12 & 11.37 & 11.97 & 62.10 & 66.53 & 3.53 & 11.77 & 90.20 & 1.43 & 63.80 \\
\hline 13. & CHFPUM-13 & 7.90 & 8.10 & 59.73 & 63.97 & 3.30 & 12.13 & 82.00 & 3.40 & 63.60 \\
\hline 14. & CHFPUM-14 & 6.70 & 9.53 & 58.83 & 62.73 & 3.63 & 10.77 & 81.40 & 1.87 & 56.80 \\
\hline 15. & CHFPUM-15 & 7.67 & 10.07 & 58.53 & 63.53 & 4.43 & 12.07 & 82.37 & 2.60 & 55.63 \\
\hline 16. & CHFPUM-16 & 7.90 & 11.73 & 59.73 & 65.00 & 4.50 & 11.37 & 85.00 & 3.57 & 64.13 \\
\hline 17. & CHFPUM-17 & 8.57 & 10.83 & 61.20 & 64.87 & 3.33 & 13.33 & 85.20 & 2.50 & 58.73 \\
\hline 18. & CHFPUM-18 & 6.83 & 10.97 & 63.43 & 70.10 & 3.30 & 11.87 & 91.10 & 2.63 & 58.00 \\
\hline 19. & CHFPUM-19 & 6.57 & 9.00 & 59.40 & 62.53 & 3.93 & 14.13 & 86.27 & 3.57 & 64.93 \\
\hline 20. & CHFPUM-20 & 6.90 & 11.10 & 62.77 & 63.73 & 3.00 & 11.20 & 82.70 & 2.00 & 68.93 \\
\hline 21. & CHFPUM-21 & 7.07 & 11.53 & 62.07 & 63.53 & 3.20 & 11.83 & 79.20 & 2.10 & 59.00 \\
\hline 22. & CHFPUM-22 & 6.37 & 12.73 & 62.43 & 66.20 & 3.73 & 12.73 & 82.20 & 2.40 & 56.93 \\
\hline 23. & CHFPUM-23 & 7.03 & 11.17 & 60.97 & 62.30 & 3.97 & 14.33 & 81.10 & 2.70 & 66.80 \\
\hline 24. & CHFPUM-24 & 6.60 & 11.67 & 62.87 & 64.63 & 2.73 & 10.43 & 81.30 & 2.13 & 63.63 \\
\hline 25. & CHFPUM-25 & 7.27 & 12.87 & 60.63 & 63.17 & 3.97 & 12.10 & 84.50 & 3.13 & 52.80 \\
\hline & Mean & 7.63 & 10.96 & 61.27 & 65.22 & 4.04 & 12.33 & 86.91 & 2.66 & 58.83 \\
\hline & CV\% & 8.74 & 10.15 & 2.09 & 2.11 & 17.93 & 10.11 & 2.76 & 9.21 & 4.29 \\
\hline & $\mathbf{S E}+\mathbf{m}$ & 0.38 & 0.64 & 0.74 & 0.79 & 0.41 & 0.71 & 1.38 & 0.14 & 1.45 \\
\hline & LSD (5\%) & 1.09 & 1.82 & 2.10 & 2.26 & 1.19 & 2.04 & 3.94 & 0.40 & 4.14 \\
\hline & Minimum & 6.3667 & 8.06 & 58.53 & 62.00 & 2.73 & 10.43 & 79.20 & 1.43 & 41.93 \\
\hline & Maximum & 11.36 & 13.63 & 63.50 & 70.73 & 6.33 & 14.33 & 103.33 & 3.73 & 68.93 \\
\hline
\end{tabular}


Table.3 Yield contributing characters of 25 genotypes of pumpkin

\begin{tabular}{|c|c|c|c|c|c|c|c|c|c|}
\hline $\begin{array}{l}\text { Sl. } \\
\text { No. }\end{array}$ & Charectes & $\begin{array}{l}\text { Equatorial } \\
\text { circumfe } \\
\text { rences }\end{array}$ & $\begin{array}{l}\text { Flesh } \\
\text { thickness }\end{array}$ & $\begin{array}{l}\text { Cavity } \\
\text { length }\end{array}$ & $\begin{array}{l}\text { Number } \\
\text { of seeds } \\
\text { per fruit }\end{array}$ & $\begin{array}{l}100 \text { seed } \\
\text { weight } \\
\text { (g) }\end{array}$ & $\begin{array}{l}\text { Average } \\
\text { fruit weight } \\
(\mathrm{kg})\end{array}$ & $\begin{array}{ll}\text { Fruit } & \text { yield } \\
\text { per } & \text { plant } \\
(\mathrm{kg}) & \end{array}$ & $\begin{array}{l}\text { Fruit yield } \\
\text { per hectare } \\
\text { (q) }\end{array}$ \\
\hline 1. & CHFPUM-1 & 37.80 & 2.77 & 18.57 & 209.67 & 9.67 & 1.57 & 4.97 & 212.20 \\
\hline 2. & CHFPUM-2 & 51.50 & 3.23 & 7.77 & 318.33 & 8.20 & 1.37 & 5.83 & 249.03 \\
\hline 3. & CHFPUM-3 & 60.00 & 4.50 & 10.17 & 213.33 & 7.57 & 2.00 & 5.70 & 210.20 \\
\hline 4. & CHFPUM-4 & 63.50 & 4.50 & 11.90 & 205.00 & 9.80 & 1.82 & 4.66 & 199.10 \\
\hline 5. & CHFPUM-5 & 51.63 & 2.77 & 5.93 & 401.00 & 8.37 & 1.60 & 2.83 & 121.07 \\
\hline 6. & CHFPUM-6 & 46.80 & 2.43 & 18.17 & 183.33 & 5.07 & 2.00 & 5.20 & 222.20 \\
\hline 7. & CHFPUM-7 & 61.00 & 3.67 & 10.23 & 301.67 & 9.23 & 1.70 & 4.23 & 180.87 \\
\hline 8. & CHFPUM-8 & 48.10 & 2.90 & 8.67 & 186.67 & 4.77 & 1.65 & 3.40 & 145.23 \\
\hline 9. & CHFPUM-9 & 52.43 & 3.87 & 7.63 & 290.00 & 5.83 & 2.15 & 5.83 & 249.23 \\
\hline 10. & CHFPUM-10 & 67.93 & 3.77 & 11.27 & 263.33 & 14.57 & 2.37 & 6.27 & 267.77 \\
\hline 11. & CHFPUM-11 & 58.81 & 2.80 & 11.53 & 226.67 & 6.97 & 2.70 & 6.03 & 257.80 \\
\hline 12. & CHFPUM-12 & 58.30 & 5.17 & 10.60 & 198.33 & 6.03 & 2.87 & 3.03 & 129.60 \\
\hline 13. & CHFPUM-13 & 63.30 & 2.83 & 11.57 & 229.33 & 10.30 & 2.07 & 5.17 & 220.73 \\
\hline 14. & CHFPUM-14 & 63.13 & 4.30 & 13.27 & 314.00 & 11.23 & 2.90 & 4.83 & 206.50 \\
\hline 15. & CHFPUM-15 & 59.30 & 3.23 & 10.53 & 236.67 & 10.37 & 2.75 & 6.43 & 274.67 \\
\hline 16. & CHFPUM-16 & 33.93 & 2.60 & 18.17 & 209.33 & 8.97 & 1.70 & 7.37 & 314.77 \\
\hline 17. & CHFPUM-17 & 61.93 & 4.77 & 8.07 & 206.00 & 12.03 & 1.77 & 4.63 & 197.97 \\
\hline 18. & CHFPUM-18 & 56.10 & 3.23 & 9.50 & 296.67 & 6.47 & 2.47 & 5.97 & 254.97 \\
\hline 19. & CHFPUM-19 & 61.30 & 2.83 & 10.50 & 218.33 & 6.23 & 2.90 & 7.07 & 301.93 \\
\hline 20. & CHFPUM-20 & 40.30 & 2.33 & 23.57 & 206.67 & 11.50 & 1.67 & 3.60 & 153.80 \\
\hline 21. & CHFPUM-21 & 64.13 & 2.93 & 10.50 & 188.67 & 7.93 & 2.50 & 3.80 & 162.33 \\
\hline 22. & CHFPUM-22 & 61.30 & 3.33 & 9.77 & 189.67 & 9.90 & 2.40 & 6.00 & 256.17 \\
\hline 23. & CHFPUM-23 & 73.80 & 3.50 & 10.77 & 134.33 & 14.37 & 3.00 & 5.77 & 246.17 \\
\hline 24. & CHFPUM-24 & 58.50 & 3.63 & 14.73 & 204.33 & 7.10 & 2.63 & 3.90 & 166.60 \\
\hline \multirow[t]{7}{*}{25.} & CHFPUM-25 & 59.43 & 3.17 & 10.83 & 296.67 & 11.03 & 2.20 & 5.73 & 244.97 \\
\hline & Mean & 56.57 & 3.40 & 11.76 & 237.12 & 8.94 & 2.18 & 5.13 & 219.16 \\
\hline & CV\% & 3.23 & 9.69 & 4.48 & 10.01 & 6.42 & 12.98 & 5.92 & 5.93 \\
\hline & $\mathrm{SE} \pm \mathrm{m}$ & 1.05 & 0.19 & 0.30 & 13.71 & 0.33 & 0.16 & 0.17 & 7.50 \\
\hline & LSD (5\%) & 3.00 & 0.54 & 0.86 & 39.00 & 0.94 & 0.46 & 0.49 & 28.48 \\
\hline & Minimum & 33.93 & 2.33 & 5.93 & 134.33 & 4.76 & 1.36 & 2.83 & 121.06 \\
\hline & Maximum & 73.80 & 5.16 & 23.56 & 401.00 & 14.56 & 3.00 & 7.36 & 314.76 \\
\hline
\end{tabular}

Fig.1 Graph showing TSS in different pumpkin genotypes (LSD at 5\% 1.21)

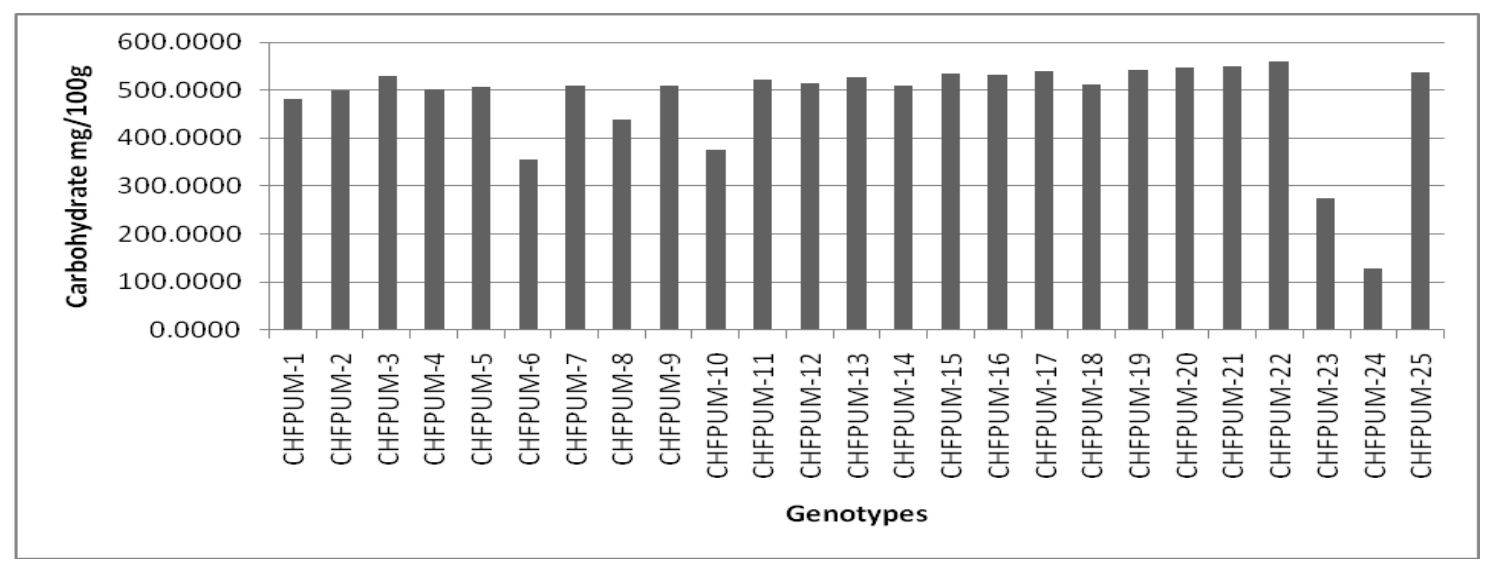


Fig.2 Carbohydrate content (mg/100g) of different genotypes of Pumpkin (LSD at 5\% 22.46)

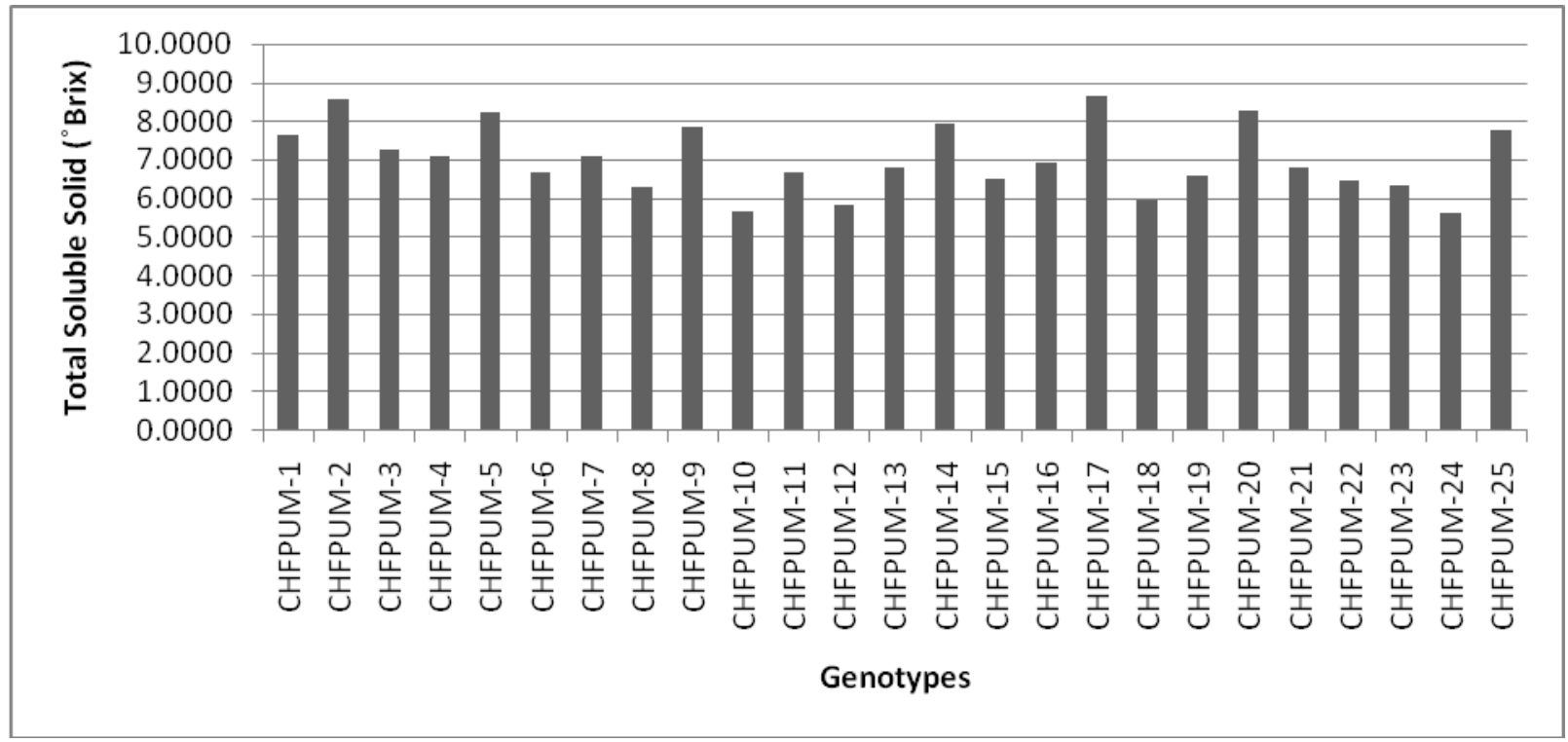

Fig.3 Graph showing vitamin-A content in different genotypes of pumpkin (LSD at 5\% 0.3)

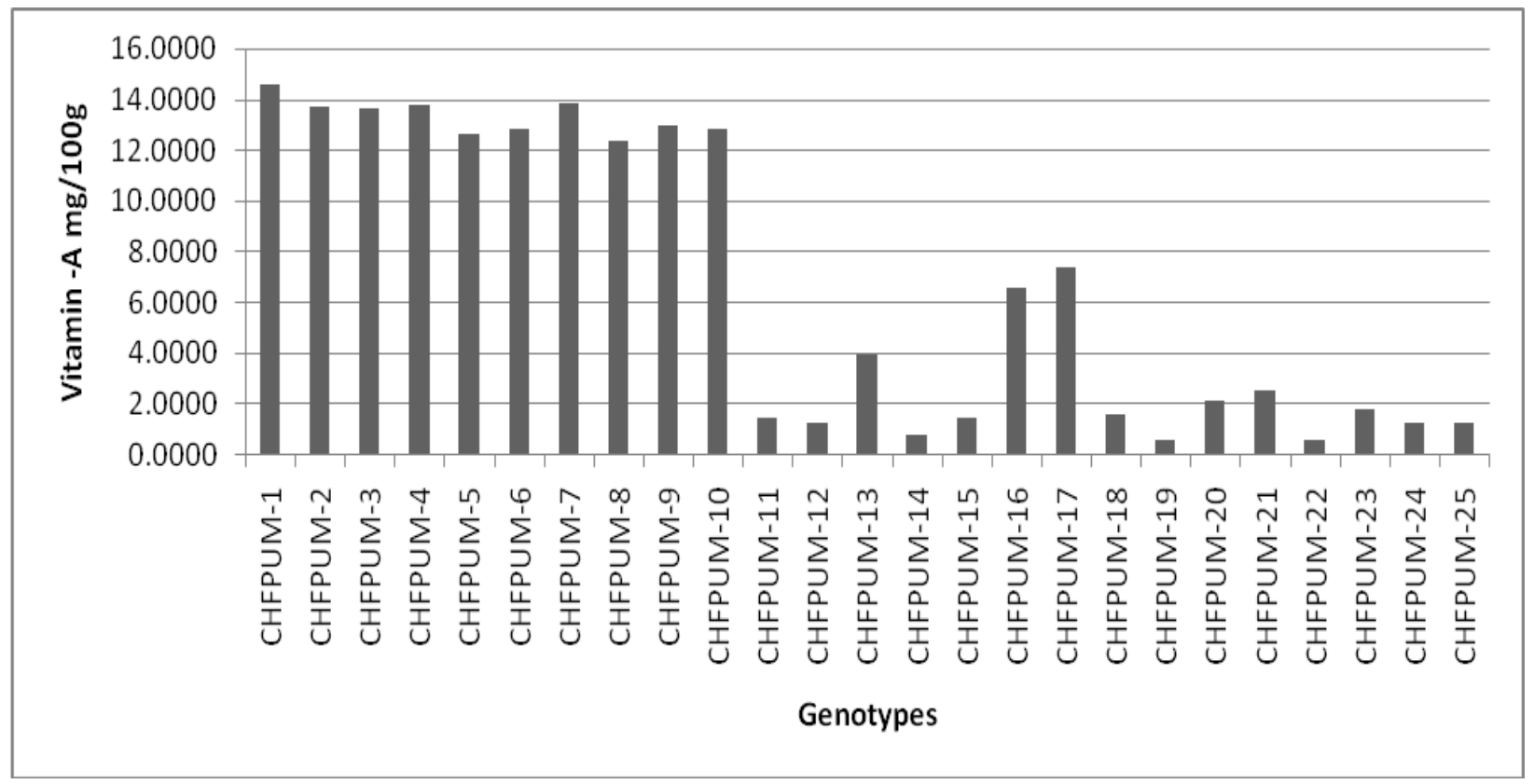

Minimum cavity length was noted in CHFPUM-5 (5.93) followed by CHFPUM-9 (7.63) and CHFPUM-2 (7.76) (Table 3). Highest number of fruits per plant was observed in genotype CHFPUM-2 (3.73) followed by CHFPUM-16 and CHFPUM-19 (Table 3$)$. The result was in conformity with Tamilselvi and Jansirani (2017). The average fruit weight of pumpkin genotype was highest recorded in genotypes CHFPUM-23 $(3.00 \mathrm{~kg})$ followed by CHFPUM-14 and CHFPUM-19 (Table 3). Same result was observed by Ahmed et al., (2011) and Venkatesan et al., (2016). Maximum fruit yield per plant was also found in genotype CHFPUM-16 (7.36 $\mathrm{kg}$ ) followed by CHFPUM-19 and CHFPUM- 
15 (Table 3) and this result was in conformity with Venkatesan et al., (2016).

The rainfall in the region is very high due to which pollen is washed and there is less pollinator activity resulting in low yield per plant. Maximum fruit yield per hectare was recorded in CHFPUM-16 (314.76) which was found statistically at par with CHFPUM-19 (Table 3).

\section{Quality characters}

Highest total soluble solids content was observed in CHFPUM-17 (8.66) followed by CHFPUM-2 and CHFPUM-5 (Fig. 1). The result was in conformity with Kumar et al., (2017), they reported a TSS range of 4.53$7.63{ }^{\circ}$ Brix in pumpkin. Maximum carbohydrate content was estimated in CHFPUM-22 (559.50 mg/100 g) it was found statically at par with CHFPUM-21, CHFPUM-20 and CHFPUM-19 (Fig. 2).

Similarly Sharma et al., (2013) reported a total sugar content of $106.58 \mathrm{mg} / 100 \mathrm{~g}$ in pumpkin.

Maximum vitamin-A content was estimated in CHFPUM-1 (14.57) followed by CHFPUM-7 and CHFPUM-4 (Fig. 3). A carotenoides content of $3.16 \mathrm{mg} / 100 \mathrm{~g}$ (Sharma et al., 2013), 2.34-14.85 mg/100 g (Pandey et al., 2003) and 3.46-8.05mg/ $100 \mathrm{~g}$ (Kumar et al., 2017) was reported in pumpkin.

Hence conclusion, on the basis of mean performance, the genotype CHFPUM-24 (Landrace from Tripura) was found good for earliness and growth. The genotype CHFPUM-16 (landrace from Imphal, Manipur) was found to be superior for the yield attributing characters while CHFPUM-1 (landrace from Pasighat, Arunachal Pradesh) was found good for fruit quality. So, these genotypes can be used as parental source in future breeding programme.

\section{Acknowledgement}

The author is thankful to the Director of ICAR-IIVR, Varanasi for providing the pumpkin varieties for this study.

\section{References}

Aboul-Nasr, M.H., Ramdan, B.R. and ElDengawy, R.A. (1997). Chemical composition of pumpkin seeds. Assiut. J. Agric. Sci., 28(1): 163-172.: pp. 7.

Ahamed, K.U. Akhter, B. Islam, M.R. Ara, N. and Humauan, M.R. (2011) an assessment of morphology and yield characteristics of pumpkin (cucurbita moschata) genotypes in northern Bangladesh. Tropical Agric. Res. Ext., 14(1): 7-11.

Bahadur, A. and Singh, K. P. (2014). Pumpkin. In: Rana, M.K. (ed.) Scientific cultivation of vegetables. Kalyani Publishers, New Delhi, pp. 209-246.

Gomez, A.K. and Gomez, A.A. (1984). Statistical Procedures for Agricultural Research. $2^{\text {nd }}$ edition. Wiley India Private Limited, Ansari road, Daryaganj, New Delhi, pp. 134-138.

Kumar, V. Mishra1, D.P. Yadav, G.C. and Dwivedi, D.K. (2017). Evaluation of F1 hybrids/genotypes of pumpkin for biochemical traits. Int.J.Curr. Microbiol.App.Sci., 6(5): 982-989.

Pandey, S. Singh, J. Upadhyay, A. K. Ram, D.and Rai, M. (2003). Ascorbate and carotenoid content in anIndian ollection of umpkin (Cucurbita moschata Duch. ex Poir.). Cucurbit Genetics Cooperative Report., 26:5153.

Rana, M.K. (2014). Pumpkin In: Scientific cultivation of vegetables. Kalyani Publishers, New Delhi, pp. 209-246. 
Sharma, S. and Rao, R. (2013). Nutritional quality characteristics of pumpkin fruit as revealed by its biochemical analysis. Int.Food Res. J. 20(5): 23092316.

Tamilselvi, N.A., Jansirani, P., Pugalendhi, L. and Nirmalakumari, A. (2012). Performance of genotypes and correlation analysis in pumpkin
(Cucurbita moschata duch.ex poir) Electron. J. Plant. Breeding., 3(4): 987- 994.

Venkatesan, K. Malleswara, B. Reddy and Senthil, N. (2016). Evaluation of Muskmelon (Cucumis melo L.) genotypes for growth, yield and quality traits. Elect. J. Plant Breeding., 7(2): 443-447..

\section{How to cite this article:}

Ramjan, Md., A.K. Pandey, Megha Raghavan, Hau Ngaih Lian and Mohd Talha Ansari. 2018. Variations in Pumpkin (Cucurbita moschata Duch. ex Poir.) Landraces for Earliness, Yield and Quality Attributes in North East India. Int.J.Curr.Microbiol.App.Sci. 7(07): 741-748. doi: https://doi.org/10.20546/ijcmas.2018.707.091 